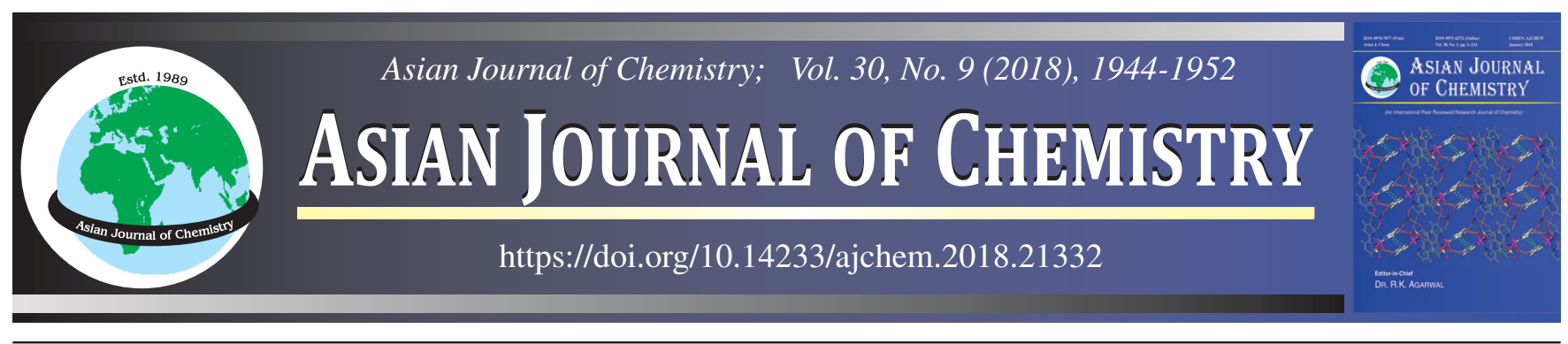

\title{
A Quest for New Antimalarial Agents with Improved Specificity Guided by Molecular Docking, 3D QSAR and Molecular Dynamics Simulation Studies
}

\author{
Janaiah Chevula, Saikrishna Balabadra, Sree Kanth Sivan and Vijullatha Manga*
}

Molecular Modeling and Medicinal Chemistry Group, Department of Chemistry, University College of Science, Osmania University, Hyderabad500007 , India

*Corresponding author: Fax: +91 40 27090020; Tel: +91 40 27682337; E-mail: vijjulathamanga@ gmail.com

Received: 13 March 2018;

Accepted: 3 July 2018;

Published online: 31 July 2018;

AJC-19000

\begin{abstract}
Chloroquine (CQ)-resistance strain of Plasmodium falciparum is posting as an alarming hitch in curing malarial disease. Efforts are on to overcome this drug resistance and to produce potential inhibitors. In this context, three-dimensional quantitative structure-activity relationship (3D-QSAR) and docking studies were extended out for recently reported 4-aminoquinoline rhodanine, 4-aminoquinoline tetrazoles, 4-anilinoquinoline triazines, 9-anilinoacridine triazines. The model generated showed good correlation coefficients $\mathrm{r}^{2}(0.961$ and 0.965$)$ and test set prediction coefficients $\mathrm{r}^{2}(0.600$ and 0.620$)$; all reinforce showed the good predictive competence of the QSAR model derived. Based on outcome of results we designed new inhibitors. These newly designed molecules were docked into active site of protein. The docking results revealed that these molecules not only interact specifically with Glu 122 in the NADH binding pocket as that of best active compound but also showed additional interactions with Leu 115. Further, molecular dynamics simulations were also carried which reinforced the docking results and showed that the newly designed molecules formed more stable complexes when compared to existing ligands. Furthermore these molecules retained interactions with active site amino acid residue Glu 122 for more percentage of simulation time which is crucial for enhancing inhibitory activity. Therefore it can be concluded that these newly designed molecules can further be modified so as to generate more potent anti malarial lead structures in near future.
\end{abstract}

Keywords: 4-Aminoquinoline hybrids, pf3D7 Strain, Molecular docking, 3D-QSAR, Molecular dynamics simulation.

\section{INTRODUCTION}

Malaria continues to be one of the major causes of morbidity even today since its discovery. It is mainly caused by Plasmodium falciparum and is prevalent in sub-tropical countries. Due to vituperative nature it drastically affected the health of the people and created a financial setback in developing countries. To ease this problem United Nation Development Program (UNDP) has mentioned this as a millennium development goal. The inefficient control of this disease is due to lack of novel anti plasmodial drugs [1], transpiring resistance [2] and slow progress in the development of new approved vaccines [3]. Further resistance of Plasmodium species towards primaquine, artemisinin and quinoline based drugs have contributed to its wide spread. Hence, there is an urgent need to develop new drugs which can help in combating malaria.

Mutations in the active sites of receptors are primarily responsible for drug resistance. Lactate dehydrogenase (LDH) enzyme of $P$. falciparum is considered to be a prospective target to fight drug resistance and increased specificity in human malaria. Lactate dehydrogenase enzyme is essential for the production of energy, it catalyzes the formation of lactate in the ultimate stage of glycolysis pathway by converting pyruvate. Plasmodium falciparum lactate dehydrogenase (pfLDH) has been chosen for the present study due to its distint active site and substrate specificity. The emergence of resistance towards commonly employed drugs like chloroquine, quinine, and mefloquine has limited 4-amino-quinoline scaffold in treatment of malaria [4]. Hence, it has essential compulsory to investigate efficacious drugs which can efficiently tackle malaria. Therefore, in present study we employed integrated molecular docking, 3D-QSAR and molecular dynamics (MD) simulation approaches on 4-aminoquinoline derivatives in order to explore the probable binding modes and derive key structural features to enhance their inhibitory activity.

This is an open access journal, and articles are distributed under the terms of the Creative Commons Attribution-NonCommercial 4.0 International (CC BY-NC 4.0) License, which allows others to copy and redistribute the material in any medium or format, remix, transform, and build upon the material, as long as appropriate credit is given and the new creations are licensed under the identical terms. 


\section{EXPERIMENTAL}

A total of 70 molecules [5-8] with known inhibition of pf $3 \mathrm{~d} 7$ strain were taken for the present study. Linux operating system was used for molecular modeling calculations. The crystal structure of pf LDH bound with chloroquine inhibitor (pdb id: 1CET) [9] was downloaded from RCSB protein data bank. Docking studies were performed using GLIDE 5.6 using Extra precision (XP) docking mode [10] active site of the receptor was made flexible by scaling the van der Waals region to 0.9 [11], this approach softens active site region [12]. The low energy conformers of the ligands were obtained by applying OPLS 2005 force field. Structures of the molecules are given in Table1 , their $\mathrm{IC}_{50}, \mathrm{pIC}_{50}$ and dock score values are given in Table- 2 . Most promising poses of ligands obtained from docking were subjected to 3D QSAR analysis $[13,14]$. CoMFA and CoMSIA studies were carried out as reported earlier [15-17] using SYBYLX -2.1 [18] by applying Gasteiger-Huckel [19] charges. CoMFA and CoMSIA models were developed using training set of 50 molecules by applying PLS analysis. Predictive ability of the models was determined using test set of 20 molecules.
Molecular dynamics simulation: Desmond 3.8 [20] was used for molecular dynamics simulations, in order to investigate and compare the binding modes of chloroquine, molecule 49 and newly designed molecule N1. Molecular dynamics simulations were carried out as per the procedure [21] and the root mean square deviations (RMSD) were examined during course of simulation and interaction diagrams of the simulation were also generated to check the stability of hydrogen bond interactions.

\section{RESULTS AND DISCUSSION}

Plasmodium falciparum CQ-sensitive inhibitors were docked into the active site (Val 26, Phe 52, Asp 53, Ile 54, Val 55, Tyr 85, Ala 98, Gly 99, Phe 100, Lys 118, Ile 119 and Glu122) of pfLDH. Dock pose of most active molecule 49 is depicted in Fig. 1.

3D QSAR analysis was performed using a set of 70 molecules which were divided into training and test set of 50 and 20, respectively. The statistical results of CoMFA and CoMSIA are summarized in Table-3. The statistical analysis yielded $\mathrm{q}_{\text {loo }}^{2}$ of 0.547

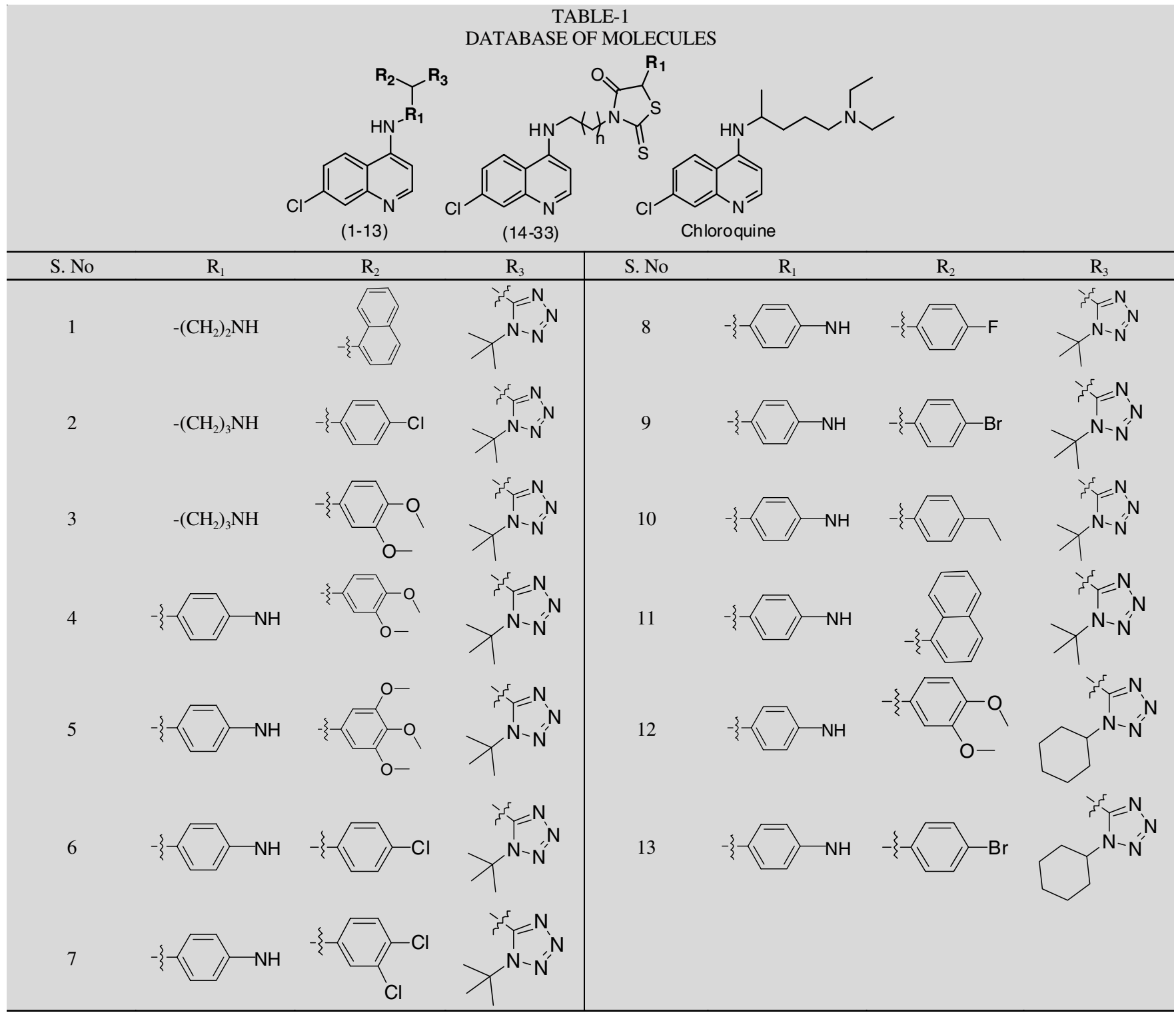




\begin{tabular}{|c|c|c|c|c|c|c|c|c|}
\hline S. No. & $\mathrm{n}$ & $\mathrm{R}_{1}$ & S. No. & $\mathrm{n}$ & $\overline{R_{1}}$ & S. No. & $n$ & $\mathrm{R}_{1}$ \\
\hline 14 & 1 & $\mathrm{H}$ & 21 & 3 & & 28 & 2 & \\
\hline 15 & 2 & $\mathrm{H}$ & 22 & 3 & & 29 & 2 & \\
\hline 16 & 3 & $\mathrm{H}$ & 23 & 2 & & 30 & 2 & \\
\hline 17 & 3 & & 24 & 2 & & 31 & 1 & \\
\hline 18 & 3 & & 25 & 2 & & 32 & 1 & \\
\hline 19 & 3 & & 26 & 2 & & 33 & 1 & \\
\hline 20 & 3 & & 27 & 2 & & & & \\
\hline
\end{tabular}

(n)




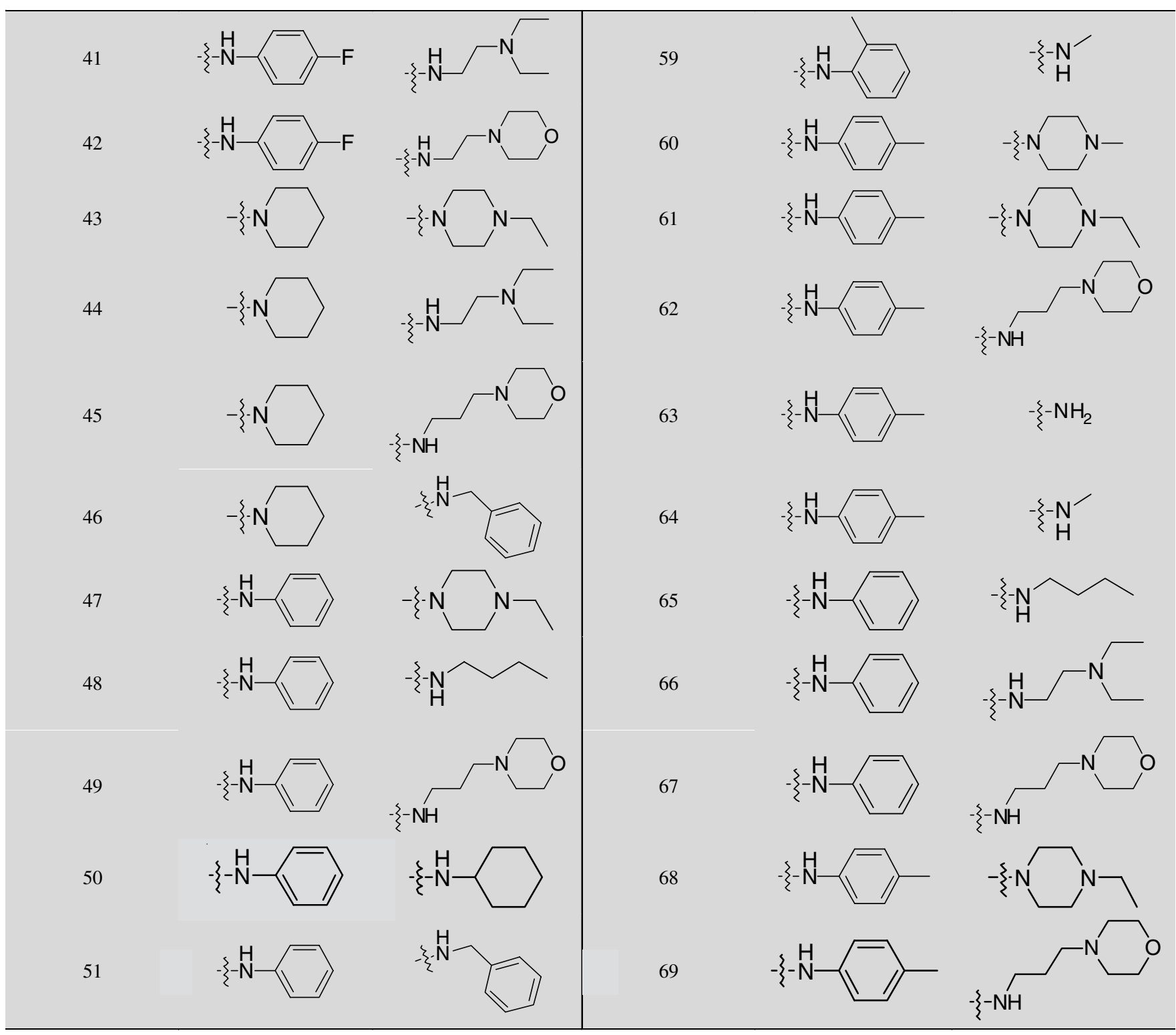

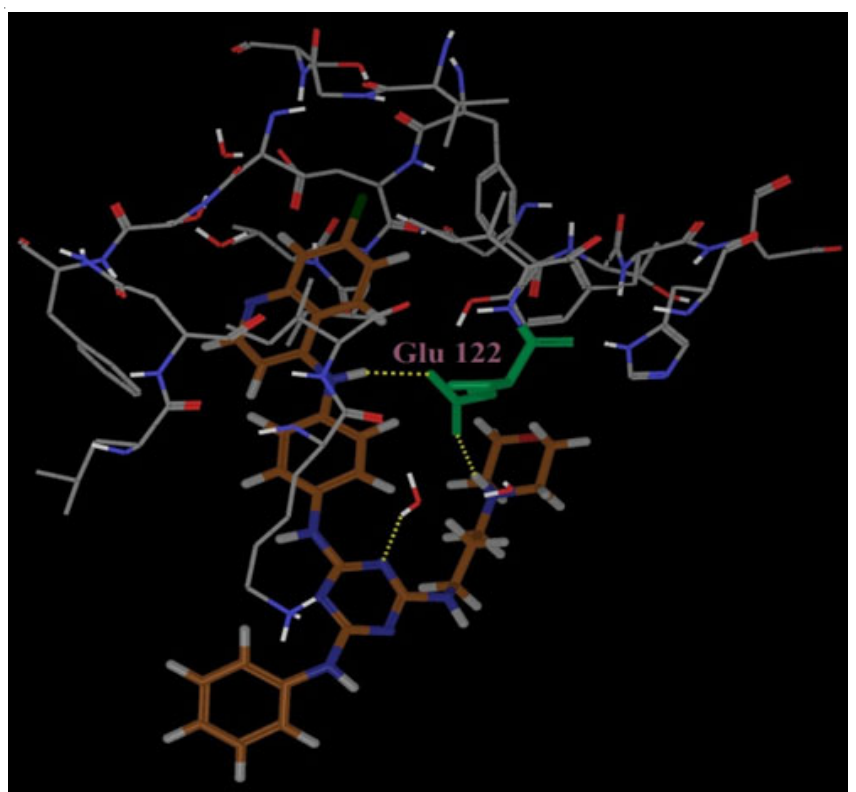

Fig. 1. Dock pose of most active molecule 49 showing H-bond interaction with Glu 122 of pfLDH and $0.533, \mathrm{r}_{\text {ncv }}^{2}$ of 0.961 and $0.965, \mathrm{r}_{\text {pred }}^{2}$ of 0.600 and 0.620 , respectively for CoMFA and CoMSIA. The results indicate good internal and external predictive ability of the models. The scatter plots of both CoMFA and CoMSIA are shown in Fig. 2.

Contour analysis: The CoMFA and CoMSIA contour maps were graphically interpreted by field contribution maps using 'STDEV COEFF' field type which represented default 80 and $20 \%$ level contribution for favored and disfavored regions.

Figs. 3a-b show contour maps of steric and electrostatic fields of both CoMFA and CoMSIA, where green and yellow contours represent favored and disfavored regions, respectively. In most active molecule 49, large green contour is seen below phenyl ring attached to triazene, suggesting substitution with bulky group at this position will increase the activity. A yellow contour near the phenyl ring attached to chloroqine suggests bulky substituents in this area will significantly decrease the biological activity. Red contour on triazene ring recommend substitution with electronegative group at this position will increase the activity. Blue contour present on phenyl ring attached 


\begin{tabular}{|c|c|c|c|c|c|c|c|c|c|c|c|}
\hline \multicolumn{12}{|c|}{$\begin{array}{c}\text { TABLE-2 } \\
\text { MOLECULES ALONG WITH THEIR IC } \text { I0 }_{50}, \mathrm{pIC}_{50}, \text { DOCK }\end{array}$} \\
\hline S. No. & $\mathrm{IC}_{50}$ & $\mathrm{pIC}_{50}$ & $\begin{array}{l}\text { Dock } \\
\text { score }\end{array}$ & $\begin{array}{c}\text { Pred } \\
\text { CoMFA }\end{array}$ & $\begin{array}{c}\text { Pred } \\
\text { CoMSIA } \\
\end{array}$ & S. No. & $\mathrm{IC}_{50}$ & $\mathrm{pIC}_{50}$ & $\begin{array}{l}\text { Dock } \\
\text { score }\end{array}$ & $\begin{array}{c}\text { Pred } \\
\text { CoMFA }\end{array}$ & $\begin{array}{c}\text { Pred } \\
\text { CoMSIA }\end{array}$ \\
\hline 1 & 120.4 & 6.919 & -5.583 & 6.926 & 6.907 & $* 36$ & 15.08 & 7.822 & -4.699 & 8.129 & 7.94 \\
\hline 2 & 81.4 & 7.089 & -6.096 & 7.067 & 7.094 & 37 & 7.95 & 8.1 & -5.572 & 8.1 & 8.11 \\
\hline 3 & 57.88 & 7.237 & -5.877 & 7.181 & 7.316 & 38 & 4.21 & 8.376 & -4.403 & 8.502 & 8.573 \\
\hline 4 & 70.58 & 7.151 & -5.019 & 7.256 & 7.185 & 39 & 9.46 & 8.024 & -6.097 & 8.054 & 8.099 \\
\hline 5 & 54.22 & 7.266 & -4.854 & 7.291 & 7.261 & 40 & 4.27 & 8.37 & -4.953 & 8.39 & 8.336 \\
\hline 6 & 10.66 & 7.972 & -5.071 & 7.725 & 7.795 & 41 & 27.88 & 7.555 & -4.862 & 7.603 & 7.579 \\
\hline 7 & 11.01 & 7.958 & -5.176 & 7.836 & 7.853 & 42 & 20.15 & 7.696 & -4.731 & 7.654 & 7.776 \\
\hline 8 & 29.52 & 7.53 & -4.933 & 7.803 & 7.798 & 43 & 29.74 & 7.527 & -4.550 & 7.476 & 7.509 \\
\hline$* 9$ & 11.78 & 7.929 & -4.960 & 7.531 & 7.579 & 44 & 38.77 & 7.412 & -4.958 & 7.404 & 7.407 \\
\hline 10 & 51.35 & 7.289 & -4.867 & 7.356 & 7.373 & 45 & 23.13 & 7.636 & -5.328 & 7.656 & 7.514 \\
\hline$* 11$ & 28 & 7.553 & -4.902 & 7.484 & 7.502 & 46 & 234.63 & 6.63 & -4.619 & 6.587 & 6.696 \\
\hline$* 12$ & 853.4 & 6.069 & -3.920 & 6.782 & 6.976 & 47 & 5.92 & 8.228 & -4.754 & 8.229 & 8.25 \\
\hline$* 13$ & 92.66 & 7.033 & -1.901 & 7.48 & 7.325 & 48 & 18.53 & 7.732 & -4.135 & 7.683 & 7.73 \\
\hline$* 14$ & 181 & 6.742 & -5.171 & 7.043 & 6.987 & 49 & 3.01 & 8.521 & -5.827 & 8.497 & 8.496 \\
\hline 15 & 200.1 & 6.699 & -5.410 & 6.883 & 6.865 & $* 50$ & 64.84 & 7.188 & -3.306 & 7.062 & 6.929 \\
\hline$* 16$ & 241.6 & 6.617 & -5.469 & 7.202 & 7.197 & $* 51$ & 33.65 & 7.473 & -4.525 & 7.7 & 7.588 \\
\hline 17 & 43.5 & 7.362 & -4.620 & 7.365 & 7.39 & 52 & 41.5 & 7.382 & -5.100 & 7.465 & 7.385 \\
\hline 18 & 54.5 & 7.264 & -4.468 & 7.26 & 7.253 & 53 & 19.03 & 7.721 & -5.487 & 7.725 & 7.704 \\
\hline$* 19$ & 93.5 & 7.029 & -4.872 & 7.446 & 7.369 & 54 & 18.85 & 7.725 & -4.916 & 7.672 & 7.692 \\
\hline$* 20$ & 35 & 7.456 & -5.345 & 7.176 & 7.182 & 55 & 26.05 & 7.584 & -3.062 & 7.548 & 7.538 \\
\hline 21 & 40 & 7.398 & -3.710 & 7.386 & 7.379 & 56 & 13.42 & 7.872 & -5.493 & 7.992 & 7.907 \\
\hline 22 & 31 & 7.509 & -3.221 & 7.479 & 7.517 & 57 & 162.99 & 6.788 & -1.681 & 6.739 & 6.798 \\
\hline 23 & 56 & 7.252 & -5.219 & 7.201 & 7.164 & 58 & 29.65 & 7.528 & -5.138 & 7.484 & 7.426 \\
\hline$* 24$ & 90.7 & 7.042 & -4.908 & 7.436 & 7.362 & 59 & 37.63 & 7.424 & -5.298 & 7.403 & 7.405 \\
\hline 25 & 23.5 & 7.629 & -4.880 & 7.633 & 7.6 & 60 & 12.44 & 7.905 & -2.719 & 7.802 & 7.89 \\
\hline$* 26$ & 96 & 7.018 & -4.013 & 7.4 & 7.375 & 61 & 11.9 & 7.924 & -4.544 & 7.836 & 7.876 \\
\hline$* 27$ & 93.9 & 7.027 & -6.268 & 7.273 & 7.22 & 62 & 7.03 & 8.153 & -5.215 & 8.259 & 8.169 \\
\hline 28 & 76.5 & 7.116 & -5.337 & 7.178 & 7.203 & $* 63$ & 11.88 & 7.925 & -4.266 & 7.58 & 7.659 \\
\hline$* 29$ & 49.3 & 7.307 & -4.953 & 7.413 & 7.126 & $* 64$ & 6.41 & 8.193 & -4.954 & 7.707 & 7.738 \\
\hline 30 & 38.2 & 7.418 & -4.721 & 7.529 & 7.325 & 65 & 26.3 & 7.58 & -3.451 & 7.622 & 7.597 \\
\hline 31 & 58.7 & 7.231 & -4.943 & 7.139 & 7.052 & 66 & 291.73 & 6.535 & -5.220 & 6.642 & 6.652 \\
\hline 32 & 42.7 & 7.37 & -3.873 & 7.303 & 7.376 & 67 & 110.67 & 6.956 & -5.559 & 6.985 & 6.911 \\
\hline 33 & 34.8 & 7.458 & -4.497 & 7.46 & 7.449 & $* 68$ & 40.95 & 7.388 & -4.779 & 7.512 & 7.68 \\
\hline$* 34$ & 22.1 & 7.656 & -3.556 & 7.655 & 7.733 & 69 & 34.56 & 7.461 & -5.849 & 7.418 & 7.445 \\
\hline$* 35$ & 6.97 & 8.157 & -4.679 & 8.389 & 8.242 & Chloroquine & 8.15 & 8.089 & -4.554 & 7.866 & 7.923 \\
\hline
\end{tabular}

*Represents test set molecules
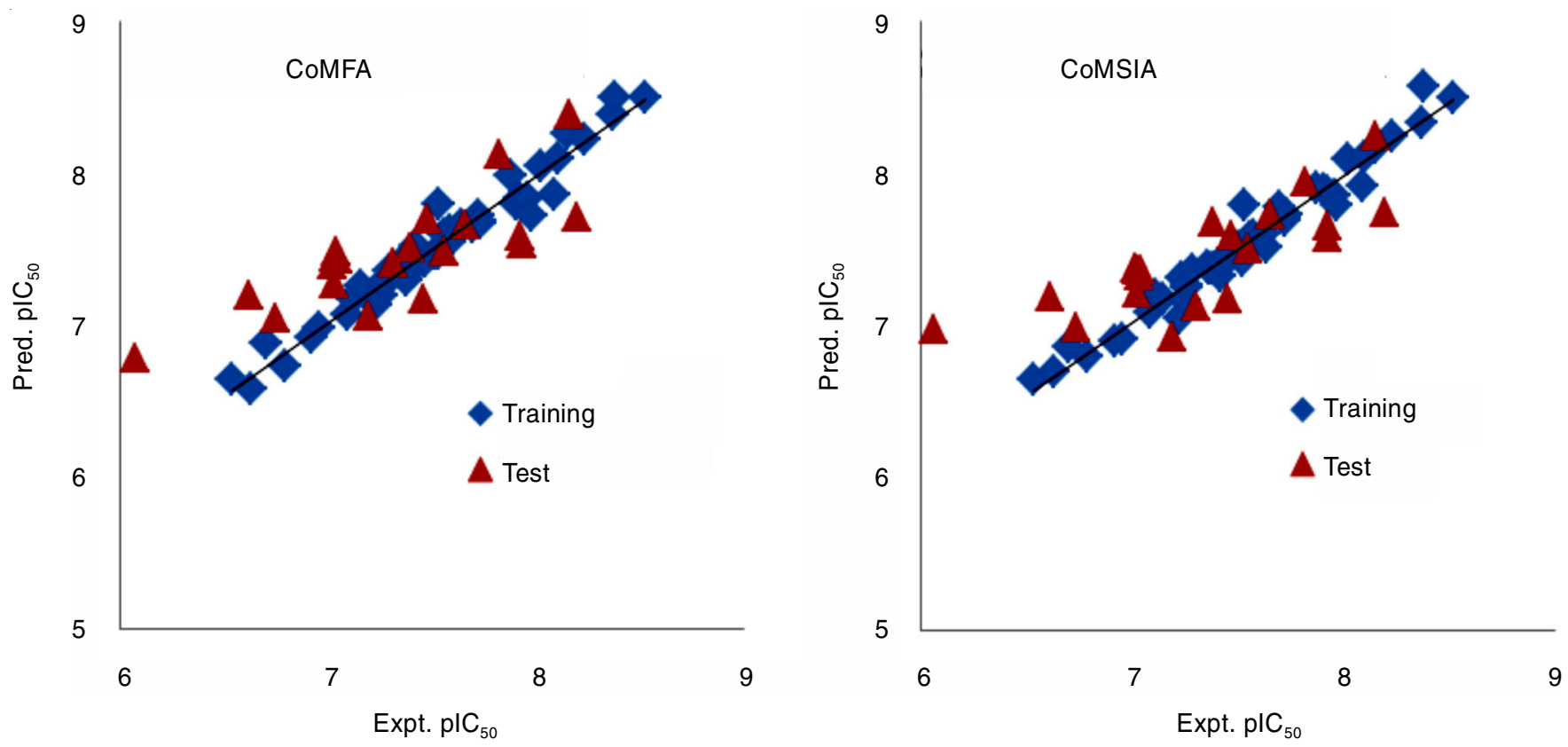

Fig. 2. Scatter plots of predicted $v s$ experimental $\mathrm{pIC}_{50}$ values (test set is represented as triangles) 
TABLE-3

STATISTICAL RESULTS FOR COMFA AND COMSIA MODELS

\begin{tabular}{lcc}
\hline \multicolumn{1}{c}{ Statistical parameters } & CoMFA model & CoMSIA model \\
\hline $\mathrm{q}^{2}$ & 0.547 & 0.533 \\
Molecules in training set & 50 & 50 \\
Molecules in test set & 20 & 20 \\
ONC & 6 & 5 \\
$\mathrm{r}_{\text {ncv }}^{2}$ & 0.961 & 0.965 \\
$\mathrm{SEE}$ & 0.095 & 0.089 \\
$\mathrm{~F}$ & 175.629 & 241.631 \\
$\mathrm{r}_{\text {pred }}^{2}$ & 0.600 & 0.620 \\
Fraction of field contributions: & & \\
$\quad$ Steric & 0.723 & 0.157 \\
$\quad$ Electrostatic & 0.277 & 0.167 \\
$\quad$ Hydrophobic & - & 0.209 \\
$\quad$ Donor & - & 0.237 \\
$\quad$ Acceptor & - & 0.230 \\
\hline
\end{tabular}

$\mathrm{q}_{\text {loo }}^{2}=$ cross-validated correlation coefficient by leave one out method, $\mathrm{r}_{\text {ncv }}^{2}=$ non cross-validated correlation coefficient, $\mathrm{ONC}=$ optimum number of components, SEE = standard error of estimate, $\mathrm{F}=$ Fisher test value, $\mathrm{r}_{\text {pred }}^{2}=$ cross-validated correlation coefficient on test set. to chloroquine signify that substitution with more electronegative groups at this position will decrease the activity.

Hydrophobic fields are depicted in Fig. 3c, where yellow and white highlighted regions represent hydrophobic and hydrophilic preferred regions, respectively. A large white contour seen close to benzene ring in the most active molecule 49 indicates that substituting bulkier hydrophilic groups will increase the activity. Hydrogen bond donor and acceptor contours in Fig. 3c show cyan and purple, magenta and red for favoured and disfavoured, respectively. For molecule 49 a donor disfavoured purple contour is observed near to $\mathrm{NH}$ of quinoline and an acceptor disfavored red contour is observed near to $\mathrm{NH}$ of benzene region indicating the decrease in activity.

Designed molecules: The information obtained from the 3D QSAR analysis is illustrated pictorially in Fig. 4, here the molecular area is divided into four regions. Region A is electronegative and hydrophilic favoured, when benzene was substituted with pyranone showed increase in predictive activity.
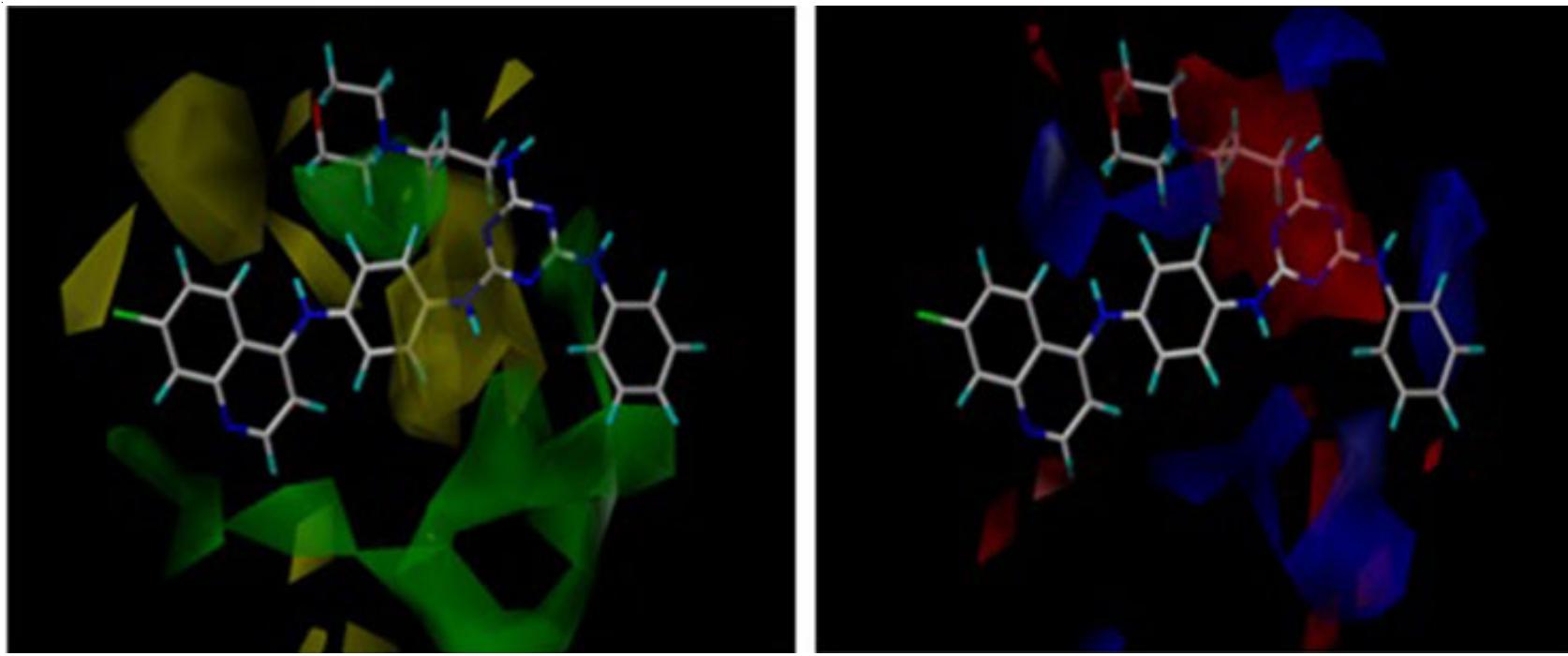

Fig. 3a. CoMFA steric (green is favored and yellow is disfavored), electrostatic (red is more negative charge and blue is more positive charge favored regions) fields contour maps for best active molecule 49
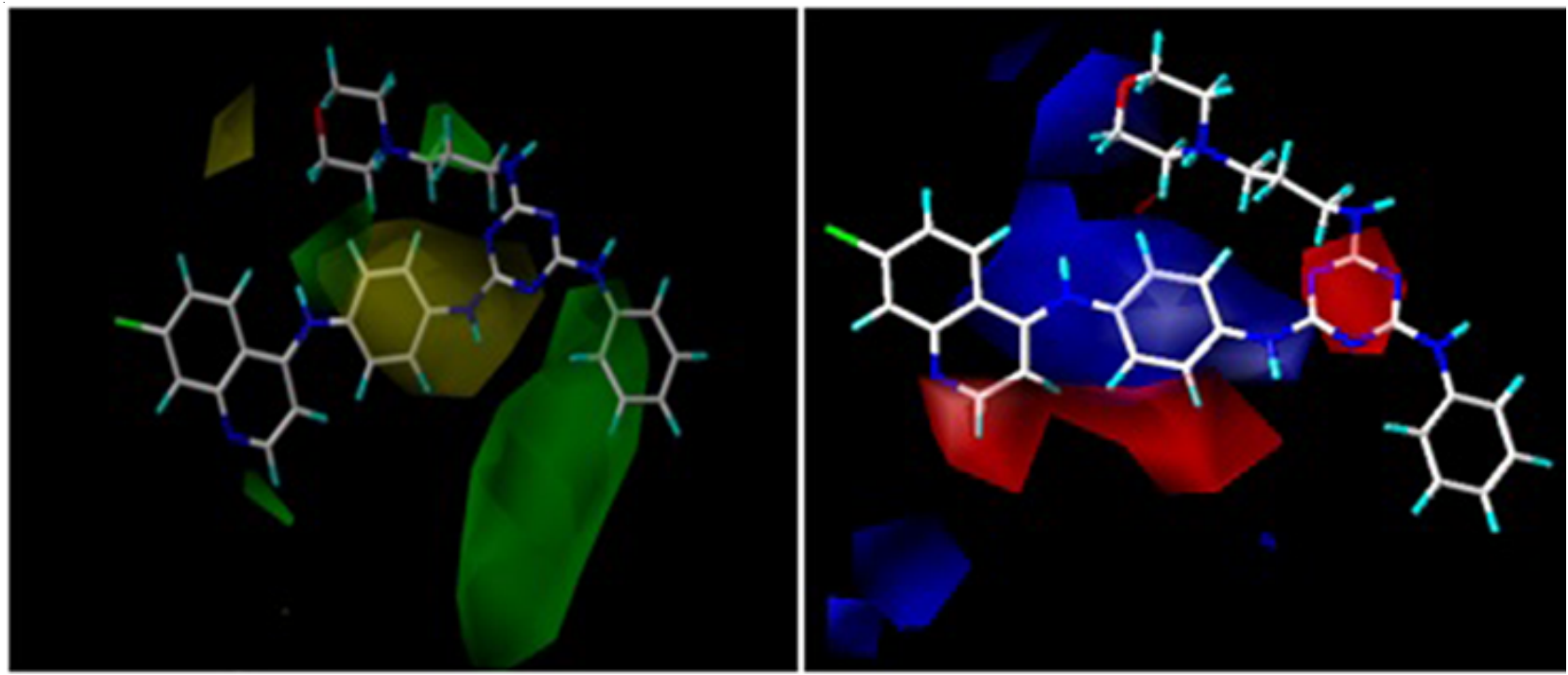

Fig. 3b. CoMSIA Steric (Green is favored and yellow is disfavored), electrostatic (red is more negative charge and blue is more positive charge favored regions) fields contour maps for best active molecule 49 

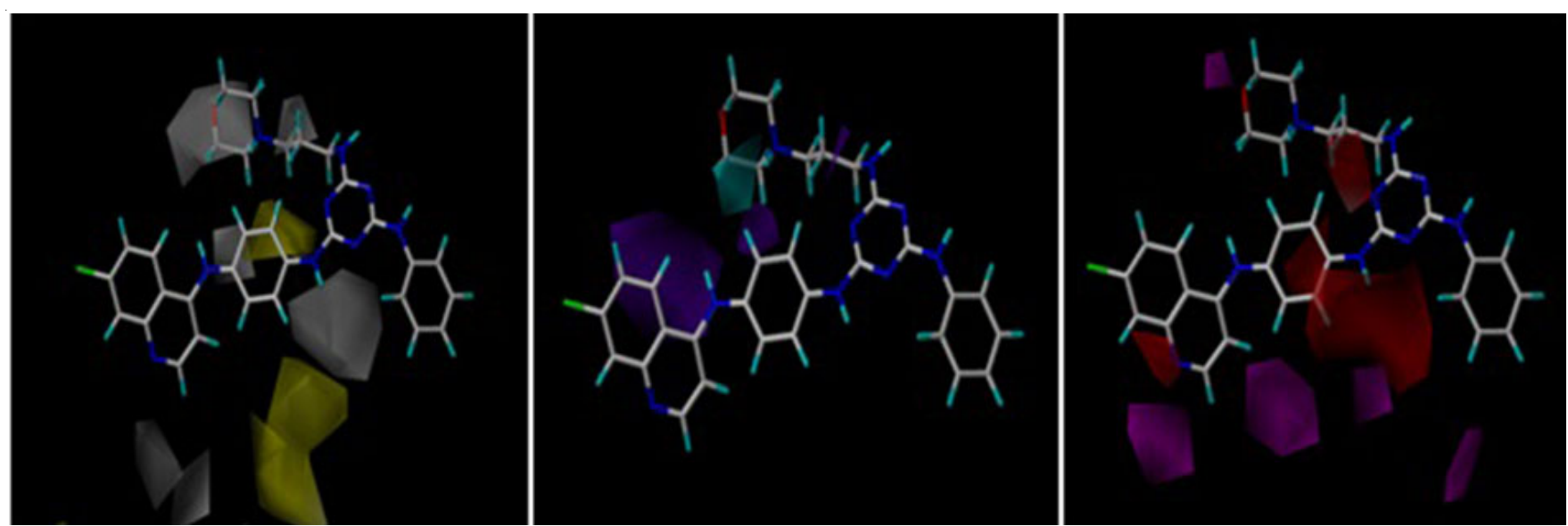

Fig. 3c. CoMSIA contour maps for best active molecule 49, Hydrophobic (white is hydrophilic and yellow is hydrophobic favored region), hydrogen bond donor (cyan is favored and purple is disfavored region), hydrogen bond acceptor (magenta and red are favored and disfavored regions respectively) fields

Region B with steric and hydrophilic favoured, substituents such as $-\mathrm{CONH}_{2}$ has increased the affinity. At region $\mathrm{C}$ steric favoured contour maps were observed, hence phenyl ring was substituted with saturated hydrocarbon chains and cyclohexyl rings to improve the activity. At region D, electrostatic favoured contour was observed, hence triazine ring was substituted with simple benzene. These entire contours were taken into consideration to substitute with desirable substituents to obtain tailored Table-4

TABLE-4

DESIGNED MOLECULES ALONG WITH DOCK SCORE AND PREDICTED ACTIVITIES<smiles>[R]c1cc([R6])cc(Nc2ccc(Nc3cc([R])nc4cc(Cl)ccc34)oc2=O)c1</smiles>

\begin{tabular}{|c|c|c|c|c|c|c|}
\hline S. No. & $\mathrm{R}_{1}$ & $\mathrm{R}_{2}$ & $\mathrm{R}_{3}$ & Dock score & Pred CoMFA & Pred CoMSIA \\
\hline N1 & $\mathrm{Me}$ & & & -6.183 & 7.989 & 8.147 \\
\hline N2 & $\mathrm{Me}$ & & & -5.637 & 8.244 & 8.120 \\
\hline N3 & $\mathrm{Me}$ & & & -5.509 & 8.137 & 8.030 \\
\hline N4 & $\mathrm{H}$ & & & -5.045 & 8.168 & 8.368 \\
\hline N5 & $\mathrm{H}$ & & & -5.965 & 8.220 & 8.156 \\
\hline N6 & $\mathrm{H}$ & & & -4.827 & 7.903 & 8.278 \\
\hline N7 & $\mathrm{H}$ & & & -5.130 & 8.212 & 7.892 \\
\hline N8 & Et & & & -5.751 & 8.206 & 8.069 \\
\hline
\end{tabular}




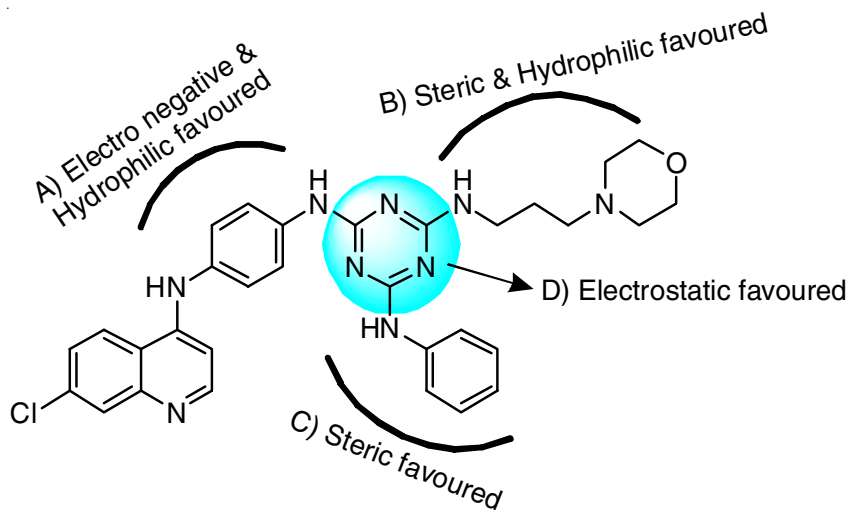

Fig. 4. Overall analysis of the contours depicting the favorable regions for substitution

molecules having high predicted activity towards Plasmodium falciparum 3D7 activity.

The designed molecules resembled best active compound in terms of interactions and showed comparable dock score with good predicted activity. Fig. 5 shows dock pose of newly designed molecule $\mathrm{N} 1$ that showed two H-bond interactions with Glu 122 and one hydrogen bond with Leu 115 in protein active site of 1CET, hence increased the binding affinity. Predicted $\mathrm{pIC}_{50}$ values were calculated and found to be better; structures of newly designed molecules and their predicted $\mathrm{pIC}_{50}$ values are given in Table-4.

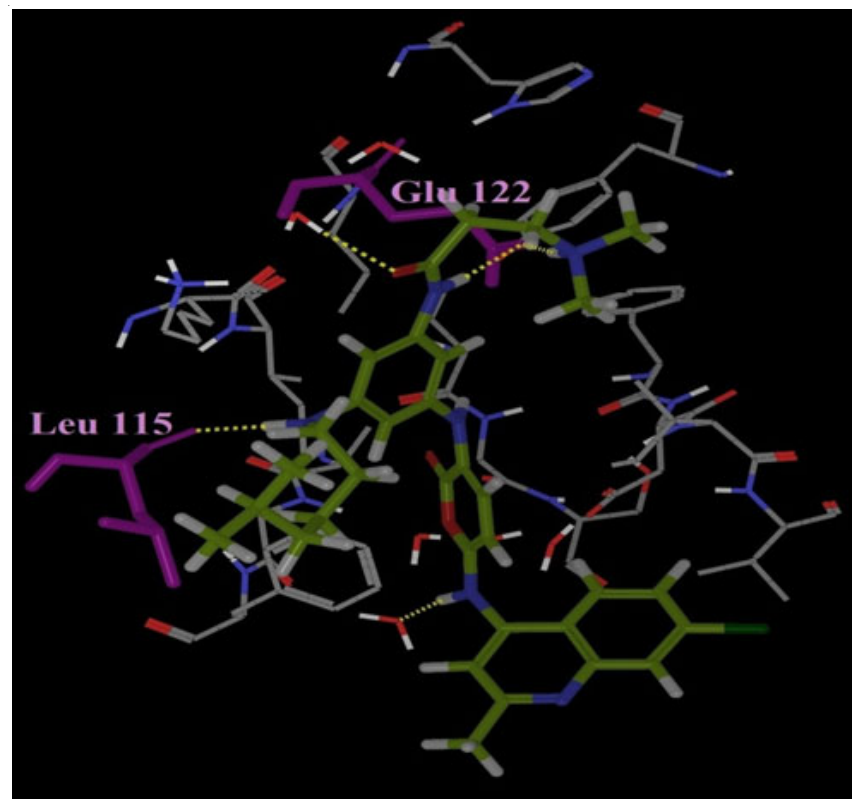

Fig. 5. Dock pose of designed molecule N1 showing two H-bond interactions with Glu 122 and one hydrogen bond with Leu 115 in the protein active site of 1CET

Molecular dynamics simulations: Molecular dynamics simulation was performed for $5 \mathrm{~ns}$ on protein ligand complex of chloroquine, molecule 49 and new molecule N1. Fig. 6 shows the RMSD of protein back bone as function of simulation time of each complex with respect to initial structure. The RMSD for chloroquine ranged from 1.056 to $2.826 \AA$ with a maximum RMSD of $3.182 \AA$ during the 5ns simulation time. RMSD for molecule 49 from the series of ligands used in 3D-QSAR studies showed RMSD of 0.984 to $2.169 \AA$ with a maximum RMSD of

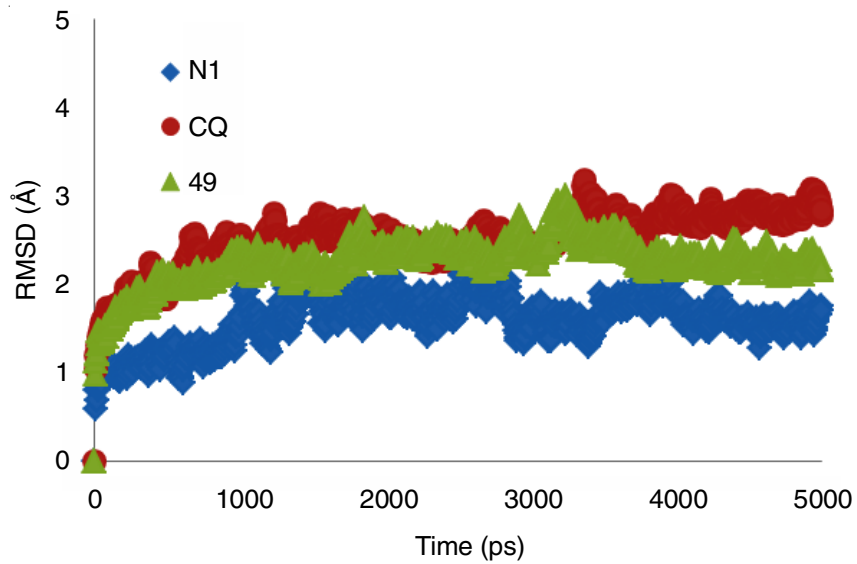

Fig. 6. RMSD of protein back bone as function of simulation time of each complex with respect to initial structure. N1 represents newly designed molecule, CQ represents Chloroquine while 49 represents best active molecule

$3.182 \AA$ during the simulation time indicating its stability which correlates to its better activity than chloroquine. Structural requirement based on 3D QSAR analysis was utilized for designing new molecules and to confirm the stability of these newly designed molecules; molecular dynamics simulations were performed for protein molecule N1 complex. The RMSD for N1 ranged from $0.595 \AA$ to $1.784 \AA$ with a maximum RMSD of $2.384 \AA$ during the $5 \mathrm{~ns}$ simulation time. The values clearly indicate that N1-Protein complex has better stability. Analysis of protein ligand interactions between pfLDH and ligands was performed to understand mode of binding and changes occurring in binding during simulations.

Glu 122 and Asp 53 are the vital residues for ligand binding. Fig. 7(a-c) shows protein ligand contact interaction over trajectory. The $y$-axis is normalized over the course of the trajectory. The chloroquine showed major hydrogen bond interaction with Asp 53 and hydrophobic interaction with Ile 54, Phe 100, and Ile 119. In case of molecule 49, it showed hydrogen bond interaction with Asp 53, but during the course of simulation it existed as water mediated bridged hydrogen bond. It retained all the hydrophobic interaction shown by chloroquine and showed more interaction with Val 26, Phe 52, Ala98, and Ile 123. The simulation interaction analysis of designed molecule $\mathrm{N} 1$ showed similar interaction with active site residues, direct hydrogen bond interaction was retained with Glu 122 for maximum duration of the simulation time and retained all other hydrophobic interaction as chloroquine. This suggests that the designed molecule $\mathrm{N} 1$ has ability to specifically bind to the conserved Glu 122 residue of pfLDH were as in all mammalian and other forms of LDH there is phenylalanine residue at this position that is not capable of forming hydrogen bonding [22].

\section{Conclusion}

To pursue novel antimalarials with improved specificity a model was developed using the in vitro antimalarial data reported, in order to design molecules with improved activity. QSAR model was statistically significant and the significance was validated. The study has provided insights to improve biological activity with the change in particular rings (anilino phenyl and triazine) in 4-aminoquinoline derivatives. Molecular docking and dynamic simulations studies highlight the exclusive binding 


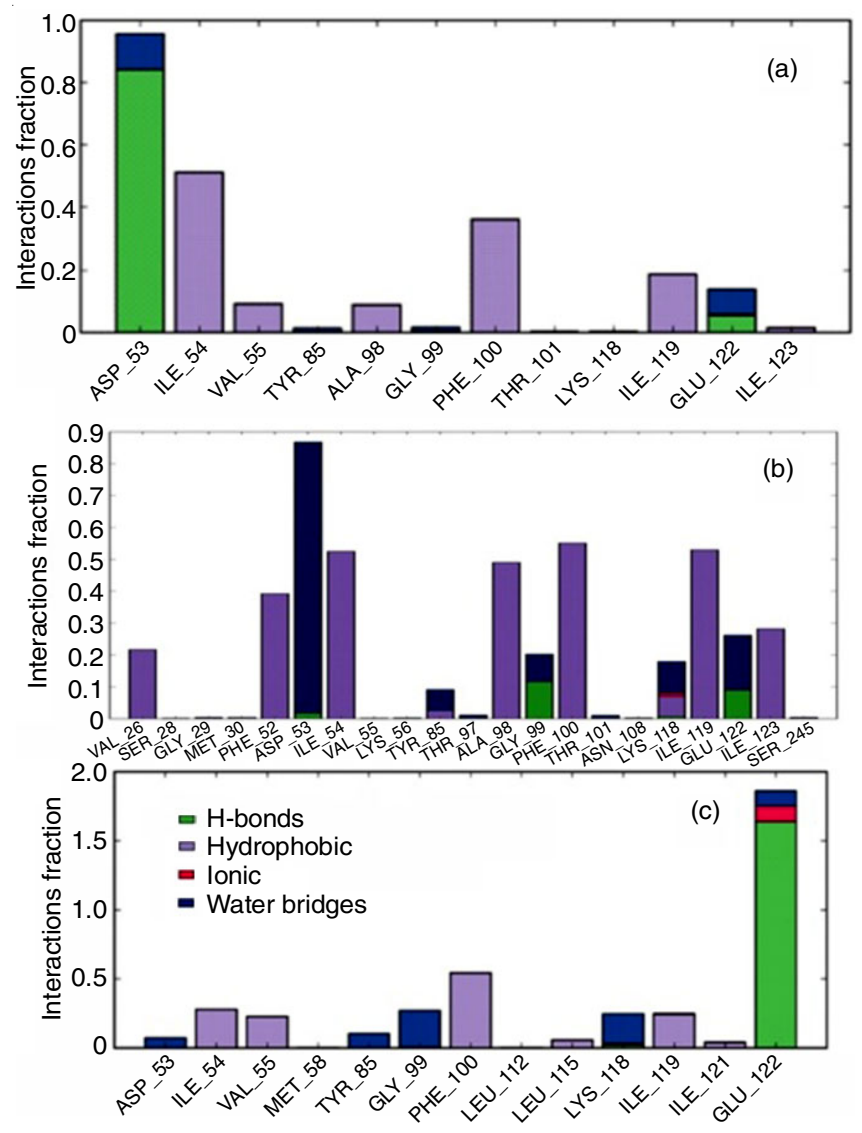

Fig. 7. Protein - ligand contact interaction over trajectory with respect (a) chloroquine, (b) molecule 49, and (c) molecule N1

signature of the ligands with the active site residue i.e. Glu122 of the target and it explains the specificity and subtle differences in their predicted $\mathrm{IC}_{50}$ values.

\section{ACKNOWLEDGEMENTS}

One of the authors, JC acknowledges UGC for financial support as research fellowship. The authors also acknowledge to Osmania University UPE funding (UPE, Focused Area of Research, No. 23/UGC/UPE/FAR/OU/2017) and the Department of Chemistry, University College of Science, Osmania University, Hyderabad, India; Tripos, Inc., USA and Schrodinger L.L.C., New York, USA are also acknowledged for the software's.

\section{CONFLICT OF INTEREST}

The authors declare that there is no conflict of interests regarding the publication of this article.

\section{REFERENCES}

1. E.H. Ekland and D.A. Fidock, Int. J. Parasitol., 38, 743 (2008); https://doi.org/10.1016/j.ijpara.2008.03.004.

2. D.A. Fidock, Nature, 465, 297 (2010); https://doi.org/10.1038/465297a.

3. M. Wisniewski and D.J. Zak, Wiad Parazytol., 56, 133 (2010).

4. R.G. Ridley, Nature, 415, 686 (2002); https://doi.org/10.1038/415686a.

5. K. Chauhan, M. Sharma, J. Saxena, S.V. Singh, P. Trivedi, K. Srivastava, S.K. Puri, J.K. Saxena, V. Chaturvedi and P.M.S. Chauhan, Eur. J. Med. Chem., 62, 693 (2013); https://doi.org/10.1016/j.ejmech.2013.01.017.

6. S. Pandey, P. Agarwal, K. Srivastava, S. RajaKumar, S.K. Puri, P. Verma, J.K. Saxena, A. Sharma, J. Lal and P.M.S. Chauhan, Eur. J. Med. Chem., 66, 69 (2013);

https://doi.org/10.1016/j.ejmech.2013.05.023.

7. A. Kumar, K. Srivastava, S. Raja Kumar, S.K. Puri and P.M.S. Chauhan, Bioorg. Med. Chem. Lett., 19, 6996 (2009); https://doi.org/10.1016/j.bmcl.2009.10.010.

8. A. Kumar, K. Srivastava, S. Raja Kumar, M.I. Siddiqi, S.K. Puri, J.K. Sexana and P.M.S. Chauhan, Eur. J. Med. Chem., 46, 676 (2011); https://doi.org/10.1016/j.ejmech.2010.12.003.

9. J.A. Read, K.W. Wilkinson, R. Tranter, R.B. Sessions and R.L. Brady, J. Biol. Chem., 274, 10213 (1999);

https://doi.org/10.1074/jbc.274.15.10213.

10. Schrödinger, LLC, New York, Glide Version 5.6, (2010).

11. R.A. Friesner, J.L. Banks, R.B. Murphy, T.A. Halgren, J.J. Klicic, D.T. Mainz, M.P. Repasky, E.H. Knoll, M. Shelley, J.K. Perry, D.E. Shaw, P. Francis and P.S. Shenkin, J. Med. Chem., 47, 1739 (2004); https://doi.org/10.1021/jm0306430.

12. D.M. Taverna and R.A. Goldstein, Proteins, 46, 105 (2002); https://doi.org/10.1002/prot.10016.

13. A.P. Zambre, A.L. Ganure, D.B. Shinde and V.M. Kulkarni, J. Chem. Inf. Model., 47, 635 (2007); https://doi.org/10.1021/ci6004367.

14. S.M. Vadlamudi and V.M. Kulkarni, Int. Electron. J. Mol. Des., 3, 586 (2004).

15. R.D. Cramer, D.E. Patterson and J.D. Bunce, J. Am. Chem. Soc., 110, 5959 (1988); https://doi.org/10.1021/ja00226a005.

16. R.D. Cramer III, J.D. Bunce, D.E. Patterson and I.E. Frank, Quant. Struct. Activ. Relat., 7, 18 (1988); https://doi.org/10.1002/qsar.19880070105.

17. G. Klebe, U. Abraham and T. Mietzner, J. Med. Chem., 37, 4130 (1994); https://doi.org/10.1021/jm00050a010.

18. Sybyl-X1,2. Tripos Associates, St. Louis (MO), www.tripos.com/sybyl, (2010).

19. J. Gasteiger and M. Marsili, Tetrahedron, 36, 3219 (1980); https://doi.org/10.1016/0040-4020(80)80168-2.

20. D.E. Shaw, DESMOND (Version 3.8), New York (2014).

21. S.R. Peddi, S.K. Sivan and V. Manga, J. Rec. Sig. Tran., 36, 488 (2016); https://doi.org/10.3109/10799893.2015.1130057.

22. J. Penna-Coutinho, W.A. Cortopassi, A.A. Oliveira, T.C. Franca and A.U. Krettli, PLoS One, 6, e21237 (2011); https://doi.org/10.1371/journal.pone.0021237. 